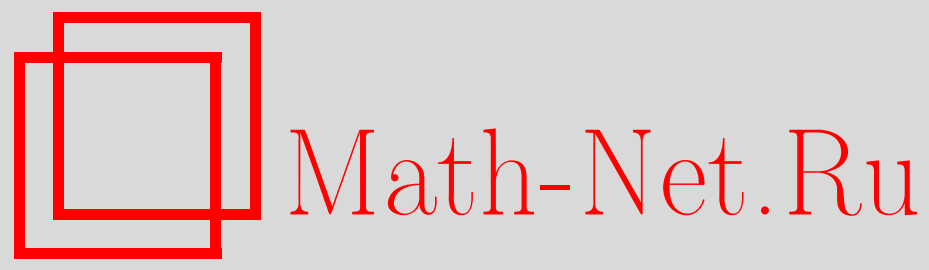

А. С. Шмелев, Функциональные модули ростков римановых метрик, Функи. анализ и его прил., 1997, том 31, выпуск 2, 58-66

DOI: https://doi.org/10.4213/faa458

Использование Общероссийского математического портала Math$\mathrm{Net.Ru}$ подразумевает, что вы прочитали и согласны с пользовательским соглашением

http://www . mathnet.ru/rus/agreement

Параметры загрузки:

IP : 54.198 .187 .58

26 апреля 2023 г., 13:20:19

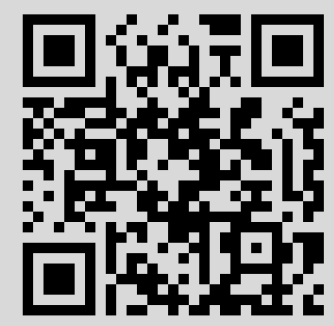


Функииональный анализ и его приложения

1997, т. 31, вып. 2, с. 58-66

УдК 515.164 .15

\section{Функциональные модули ростков римановых метрик}

(C) 1997. А. С. Шмелев

\section{Введение}

В работе рассматривается пространство $\mathrm{F}$ струй римановых метрик в точке 0 , на котором естественным образом (заменами координат) действует группа $\operatorname{Diff}\left(\mathbb{R}^{n}, 0\right)$ формальных диффеоморфизмов, сохраняющих точку 0. Пространство орбит этого действия называется пространством модулей струй римановых метрик. Если зафиксировать некоторые локальные координаты $x_{1}, \ldots, x_{n}$ в окрестности рассматриваемой точки, то струя римановой метрики будет задана симметричной матрицей $G=\left(g_{i j}\right)$, где $g_{i j}(x)$ - формальные степенные ряды, а действие группы $\operatorname{Diff}\left(\mathbb{R}^{n}, 0\right)$ на $\mathrm{F}$ будет состоять в преобразовании матрицы $G$ как матрицы симметрической формы при замене локальных координат:

$$
G \mapsto J(\varphi)^{T} G J(\varphi), \quad G \in \mathrm{F}, \varphi \in \operatorname{Diff}\left(\mathbb{R}^{n}, 0\right),
$$

где $J(\varphi)$ - якобиан формального диффеоморфизма $\varphi$.

Оказывается, что в любой размерности можно предъявить нормальную форму струи римановой метрики. Структура такой нормальной формы описывается следующей основной теоремой.

ТЕОРема. Струю римановой метрики можно привести формальным диффеоморфизмом к виду, в котором метрические коэффичиенты представляются в виде конечной суммы:

$$
g_{i j}(x)=\delta_{i j}+\sum p_{i j}^{I}(x) \varphi_{i j}^{I}(x),
$$

где сумма берется по всем подмножествам $I \subset\{1, \ldots, n\}$, не содержащимсл ни в одном из взаимно дополнительных подмножеств $I_{i j}=\{i, \ldots, j-1\}$ u $\{1, \ldots, n\} \backslash I_{i j}$, функция $\varphi_{i j}^{I}(x)$ представляет собой формальный степенной рлд по переменным $x_{i}$, где $i \in I$, а моном $p_{i j}^{I}(x)$ имеет вид

$$
p_{i j}^{I}(x)=\prod_{k \in I} x_{k} .
$$

Функции $\varphi_{i j}^{I}(x)$ называются функциональными модулями струй римановых метрик. В частности, имеется лишь конечное число функциональных модулей. Разумеется, как число функциональных модулей, так и их вид (от какого числа

* Работа выполнена при финансовой поддержке РФФИ, грант №96-01-01104, а также INTAS, грант №4373. 
каких переменных они зависят) зависит от способа приведения к нормальной форме.

Вопрос о конечности числа функциональных модулей естественных дифференциально-геометрических структур был поставлен В. И. Арнольдом в [1]. Если рассматривать пространство $k$-струй римановых метрик, на котором естественно действует группа $\operatorname{Diff}\left(\mathbb{R}^{n}, 0\right)$, то пространство орбит такого действия конечномерно. Обозначим его размерность через $a_{k}$ и составим ряд Пуанкаре

$$
p(t)=a_{0}+\sum_{k=1}^{\infty}\left(a_{k}-a_{k-1}\right) t^{k} .
$$

В работе [1] был поставлен следующий вопрос. Верно ли, что для естественных дифференциально-геометрических структур ряд Пуанкаре является рациональной функцией? Еще в работах классиков, таких, как Трессе [2], изучались вопросы о конечной порожденности алгебр дифференциальных инвариантов геометрических структур. Конечная порожденность алгебры дифференциальных инвариантов влечет за собой рациональность ряда Пуанкаре. В [3] были явно вычислены ряды Пуанкаре в случае римановых, келеровых и гиперкелеровых структур, и оказалось, что во всех трех случаях они рациональны. Следующее представление ряда Пуанкаре является следствием предъявленной выше нормальной формы струи римановой метрики.

ТеоремА. Ряд Пуанкаре пространства модулей струй римановых метрик в $\mathbb{R}^{n}$ имеет вид

$$
p(t)=t^{2} q(t)+\sum_{r=2}^{n} \frac{a_{r} t^{r}}{(1-t)^{r}}
$$

әде $q(t)$ - некоторьй полином с неположительньми коэффициентами,

$$
a_{r}=\frac{n(n-1)}{2}\left(\begin{array}{l}
n \\
r
\end{array}\right)-n \sum_{k=r}^{n-1}\left(\begin{array}{l}
k \\
r
\end{array}\right), \quad r=2, \ldots, n .
$$

Автор благодарен В. И. Арнольду за внимание к работе, а также А. М. Лукацкому и А. Г. Хованскому за полезные обсуждения.

\section{$\S 1$. Функциональные модули в пространстве струй римановых метрик}

Пусть $\mathrm{F}$ - пространство струй, а $\mathrm{F}^{k}-$ пространство $k$-струй римановых метрик в точке $0 \in \mathbb{R}^{n}$. Имеют место следующие естественные проекции

$$
\mathrm{F}^{0} \stackrel{\pi_{1}}{\longleftarrow} \mathrm{F}^{1} \stackrel{\pi_{2}}{\longleftarrow} \mathrm{F}^{2} \stackrel{\pi_{3}}{\longleftarrow} \mathrm{F}^{3} \longleftarrow \cdots
$$

сопоставляющие $k$-струе ее $(k-1)$-струю.

На пространствах $\mathrm{F}$ и $\mathrm{F}^{k}$ действует заменами координат группа $\operatorname{Diff}\left(\mathbb{R}^{n}, 0\right)$, коммутируя с проекциями $\pi_{k}$. Если $\varphi(x)=\left(\varphi_{1}(x), \ldots, \varphi_{n}(x)\right), \varphi \in \operatorname{Diff}\left(\mathbb{R}^{n}, 0\right)$, TO

$$
G \stackrel{\varphi^{*}}{\longrightarrow} J(\varphi)^{T} G J(\varphi), \quad G \in \mathrm{F}, \quad j^{k} G \stackrel{\varphi^{*}}{\longrightarrow} j^{k}\left(J(\varphi)^{T} G J(\varphi)\right), \quad j^{k} G \in \mathrm{F}^{k},
$$


где $J(\varphi)$ есть матрица Якоби диффеоморфизма $\varphi$.

Пусть $\mathrm{F}_{1}$ - касательное пространство к орбите единичной матрицы $E$ (евклидовой метрики) в точке $E$. Обозначим через $M_{G}$ орбиту элемента $G$. Всякая трансверсаль $\mathrm{T} \mathrm{F}_{1}$ в пространстве $T_{E} \mathrm{~F}$ симметричных функциональных матриц (не обязательно невырожденных) трансверсально пересекается со всеми орбитами группы $\operatorname{Diff}\left(\mathbb{R}^{n}, 0\right)$ на $\mathrm{F}$, достаточно близкими к орбите единичной матрицы.

Лемма 1.1. Если трансверсаль $\mathrm{T}$ такова, ито $T_{E} \mathrm{~F}=\mathrm{F}_{1} \oplus \mathrm{T}$, m.e. $\mathrm{T} \cap \mathrm{F}_{1}=\{0\}$, то $\mathrm{T}$ пересекается со всеми достаточно близкими $\kappa M_{E}$ орбитами по конечномерному многообразию размерности $\leqslant n(n-1) / 2$.

ДоказАтЕльство. Пересечение $M_{E} \cap \mathrm{T}=\{E\}$ - нульмерное многообразие. Стабилизатор элемента $E$ есть $O(n) \subset \operatorname{Diff}\left(\mathbb{R}^{n}, 0\right)$, стабилизатор струи общего положения - это единичная подгруппа $\{\mathrm{id}\}$, а стабилизатор струи, не являющейся струей общего положения, - подгруппа Ли в $\operatorname{Diff}\left(\mathbb{R}^{n}, 0\right)$ размерности, не превосходящей $n(n-1) / 2$ [4]. Следовательно, касательное пространство $T_{G} M_{G}$ в точке $G \in \mathrm{T} \cap M_{G}$, изоморфное факторпространству пространства $T_{\mathrm{id}} \operatorname{Diff}\left(\mathbb{R}^{n}, 0\right)$ векторных полей по конечномерному касательному подпространству к стабилизатору элемента $G$, представляется в виде прямой суммы пространства, трансверсального к Т, и конечномерного подпространства в $T_{G} \mathrm{~T}$, имеющего размерность, не превосходящую $n(n-1) / 2$.

Ниже будет доказано (теорема 1.5), что трансверсаль Т можно выбрать таким образом, что для любого $G \in \mathrm{T}$ существует набор функций $\varphi_{1}, \ldots, \varphi_{m}$, каждая из которых зависит от некоторого подмножества множества переменных $x_{1}, \ldots, x_{n}$, а элементами матрицы $G$ являются конечные суммы этих функций, умноженных на некоторые фиксированные мономы. И наоборот, любому набору таких функций $\varphi_{1}, \ldots, \varphi_{m}$ соответствует элемент $G \in \mathrm{T}$.

ОПрЕДЕЛЕНИЕ 1.2. Функции $\varphi_{1}, \ldots, \varphi_{m}$ называются функциональными модулями струй римановых метрик, а $G=G\left(\varphi_{1}, \ldots, \varphi_{m}\right) \in \mathrm{T}$ называется нормальной формой.

ЗАмЕчАНИЕ 1.3. Выбор такой трансверсали Т, вообще говоря, неоднозначен. При другом выборе трансверсали Т получится другая нормальная форма с другим набором функциональных модулей.

ЗАмЕчАниЕ 1.4. Нормальная форма, соответствующая трансверсали $\mathrm{T}$, представляет струи римановой метрики не однозначно, а лишь с точностью до некоторого конечномерного многообразия, т. е. каждой $\operatorname{Diff}\left(\mathbb{R}^{n}, 0\right)$-орбите соответствует конечномерное подмногообразие в Т, оценка размерности которого дана в лемме 1.1.

ТЕоремА 1.5. Нормальная форма Т струи римановой метрики может бъть вилбрана такой, что $\mathrm{T}=\bigoplus_{1 \leqslant i \leqslant j \leqslant n} \mathrm{~T}_{i j}$, аде $\mathrm{T}_{i j}-$ пространство формальных бесконечных линейных комбинаиий элементов подмножеств

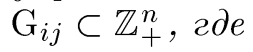

$$
\mathrm{G}_{i j}=\mathbb{Z}_{+}^{I} \backslash\left(A_{i j} \cup B_{i j}\right),
$$

$A_{i j}, B_{i j}$ - координатнье подполугруппьи вида

$$
\begin{aligned}
& A_{i j}=\left\{\left(m_{1}, \ldots, m_{n}\right) \mid m_{i}=0, i \notin\{i, \ldots, j-1\}\right\}, \\
& B_{i j}=\left\{\left(m_{1}, \ldots, m_{n}\right) \mid m_{i}=0, i \notin\{i, \ldots, i-1, j, j+1, \ldots, n\}\right\} .
\end{aligned}
$$


В частности, при $i=j$ имеем $A_{i i}=\mathbb{Z}_{+}^{n}, B_{i i}=0, \mathrm{G}_{i i}=\varnothing u \mathrm{~T}_{i i}=\{0\}$.

ДокАЗАтЕльство. Касательное пространство $\mathrm{F}_{1}$ к орбите $M_{E}$ евклидовой метрики $E=\left(\delta_{i j}\right)$ состоит из симметричных матриц, имеющих вид

$$
h_{i j}=\left(L_{v} E\right)_{i j}=\sum_{k}\left(\frac{\partial \delta_{i j}}{\partial x_{k}} v_{k}+\delta_{k j} \frac{\partial v_{k}}{\partial x_{i}}+\delta_{i k} \frac{\partial v_{k}}{\partial x_{j}}\right)=\frac{\partial v_{j}}{\partial x_{i}}+\frac{\partial v_{i}}{\partial x_{j}},
$$

где $L_{v}$ - производная Ли вдоль векторного поля $\mathbf{v}=\left(v_{1}, \ldots, v_{n}\right)$.

Покажем, что для каждого $B \in T_{E} \mathrm{~F}$ существует однозначное разложение $B=H+T$, где $H \in \mathrm{F}_{1}, T \in \mathrm{T}$, т. е.

$$
b_{i j}=\frac{\partial v_{j}}{\partial x_{i}}+\frac{\partial v_{i}}{\partial x_{j}}+t_{i j}
$$

для некоторого векторного поля $\mathbf{v}=\left(v_{1}, \ldots, v_{n}\right)$.

Пусть $T=\left(t_{i j}\right) \in \mathrm{T}$. Тогда $t_{i i}=0, i=1, \ldots, n$, а компоненты векторного поля $\mathbf{v}$ имеют вид

$$
\begin{aligned}
& v_{i}\left(x_{1}, \ldots, x_{n}\right) \\
& \quad=\frac{1}{2} \int_{0}^{x_{i}} b_{i i}\left(x_{1}, \ldots, x_{i-1}, t, x_{i+1}, \ldots, x_{n}\right) d t+u_{i}\left(x_{1}, \ldots, \hat{x}_{i}, \ldots, x_{n}\right),
\end{aligned}
$$

где «крышка» означает, что переменная $x_{i}$ отсутствует, a $u_{i}$ - произвольные формальные степенные ряды. Следовательно,

$$
t_{i j}=b_{i j}^{1}-\frac{\partial u_{j}}{\partial x_{i}}-\frac{\partial u_{i}}{\partial x_{j}}
$$

где

$$
b_{i j}^{1}=b_{i j}-\frac{1}{2} \int \frac{\partial b_{j j}}{\partial x_{i}} d x_{j}-\frac{1}{2} \int \frac{\partial b_{i i}}{\partial x_{j}} d x_{i} .
$$

Формальные степенные ряды $u_{i}, i=1, \ldots, n$, однозначно определяются из последнего уравнения и из условий $t_{i j} \in \mathrm{T}_{i j}$. Действительно, из уравнений

$$
\begin{gathered}
t_{i, i+1}=b_{i, i+1}^{1}-\frac{\partial u_{i+1}}{\partial x_{i}}-\frac{\partial u_{i}}{\partial x_{i+1}}, \quad i=1, \ldots, n-1, \\
t_{1 n}=t_{n 1}=b_{n 1}^{1}-\frac{\partial u_{1}}{\partial x_{n}}-\frac{\partial u_{n}}{\partial x_{1}}
\end{gathered}
$$

и из условий $t_{i, i+1} \in \mathrm{T}_{i, i+1}, t_{n 1} \in \mathrm{T}_{1 n}$ степенные ряды $u_{i}$ определяются однозначно с точностью до слагаемого $u_{i}^{1}$, являющегося формальным степенным рядом, не зависящим от $x_{i}$ и $x_{\tau(i)}$, где $\tau(i)=i+1$ при $i=1, \ldots, n-1$ и $\tau(n)=1$. Рассмотрим уравнения

$$
t_{i, i+2}=b_{i, i+2}^{2}-\frac{\partial u_{i+2}^{1}}{\partial x_{i}}-\frac{\partial u_{i}^{1}}{\partial x_{i+2}}, \quad i=1, \ldots, n-2,
$$

$t_{1, n-1}=t_{n-1,1}=b_{n-1,1}^{2}-\frac{\partial u_{1}^{1}}{\partial x_{n-1}}-\frac{\partial u_{n-1}^{1}}{\partial x_{1}}, \quad t_{2 n}=t_{n 2}=b_{n 2}^{2}-\frac{\partial u_{2}^{1}}{\partial x_{n}}-\frac{\partial u_{n}^{1}}{\partial x_{2}}$ 
где в $b_{i j}^{2}$ отнесены как мономы, входящие в $b_{i j}^{1}$, так и мономы, входящие в ряд $\partial u_{j} / \partial x_{i}+\partial u_{i} / \partial x_{j}$, определенный на предыдущем шаге однозначно с точностью до слагаемого $\partial u_{j}^{1} / \partial x_{i}+\partial u_{i}^{1} / \partial x_{j}$. Из этих уравнений и из условий $t_{i, i+2} \in \mathrm{T}_{i, i+2}, t_{n-1,1} \in \mathrm{T}_{1, n-1}, t_{n 2} \in \mathrm{T}_{2 n}$ степенные ряды $u_{i}^{1}$ определяются однозначно с точностью до слагаемого $u_{i}^{2}$, являющегося формальным степенным рядом, не зависящим от $x_{i}, x_{\tau(i)}, x_{\tau^{2}(i)}$. Продолжая эту процедуру, на последнем $(n-1)$-м шаге получим следующие уравнения:

$$
\begin{gathered}
t_{1 n}=b_{1 n}^{n-1}-\frac{\partial u_{n}^{n-2}}{\partial x_{1}}-\frac{\partial u_{1}^{n-2}}{\partial x_{n}} \\
t_{i, i+1}=t_{i+1, i}=b_{i+1, i}^{n-1}-\frac{\partial u_{i}^{n-2}}{\partial x_{i+1}}-\frac{\partial u_{i+1}^{n-2}}{\partial x_{i}}, \quad i=1, \ldots, n-1,
\end{gathered}
$$

где $u_{i}^{n-2}$ — формальные степенные ряды, не зависящие от $x_{i}, x_{\tau(i)}, \ldots, x_{\tau^{n-2}(i)}$, т. е. $u_{i}^{n-2}$ - формальный степенной ряд по $x_{\tau^{n-1}(i)}=x_{\tau^{-1}(i)}$. Из этих уравнений и из условий $t_{1 n} \in \mathrm{T}_{1 n}$ и $t_{i+1, i} \in \mathrm{T}_{i, i+1}, i=1, \ldots, n-1$, степенные ряды $u_{i}^{n-2}$ определяются однозначно с точностью до членов нулевой степени. Из условия $v_{i}(0)=0$ вытекает, что $u_{i}(0)=0$ и что свободный член ряда $u_{i}^{n-2}$ равен нулю. Таким образом, доказано, что для каждого элемента $B \in T_{E} \mathrm{~F}$ существует однозначное разложение $B=H+T$, где $H \in \mathrm{F}_{1}, T \in \mathrm{T}$.

СЛЕДСТВИЕ 1.6. Струю римановой метрики в $\mathbb{R}^{n}$ можно привести формальньлм диффеоморфизмом к виду $g_{i j}=\delta_{i j}+f_{i j}$, где функиия $f_{i j}, 1 \leqslant i \leqslant$ $j \leqslant n$, обрашается в нуль на паре координатных плоскостей

$$
\begin{aligned}
a_{i j} & =\left\{x_{1}=\cdots=x_{i-1}=x_{j}=x_{j+1}=\cdots=x_{n}=0\right\}, \\
b_{i j} & =\left\{x_{i}=\cdots=x_{j-1}=0\right\} .
\end{aligned}
$$

В иастности, при $i=j$ имеем $a_{i i}=\{0\}, b_{i i}=\mathbb{R}^{n}$, m. e. $g_{i i}=1, i=1, \ldots, n$.

Для каждого подмножества $I \subset\{1, \ldots, n\}$ обозначим через $\mathbb{Z}_{+}^{I}$ координатную подполугруппу $\left\{\left(m_{1}, \ldots, m_{n}\right) \mid m_{i}=0, i \notin I\right\}$ в $\mathbb{Z}_{+}^{n}$. Подполугруппа $\mathbb{Z}_{+}^{I}$ изоморфна $\mathbb{Z}_{+}^{|I|}$, где $|I|$ есть мощность подмножества $I$. При $I=\varnothing$ имеем $\mathbb{Z}_{+}^{\varnothing}=\{(0, \ldots, 0)\}$.

Лемма 1.7. Полугруппа $\mathbb{Z}_{+}^{n}$ распадается в объединение $2^{n}$ непересекающихся сдвинутых координатных подполугрупп:

$$
\mathbb{Z}_{+}^{n}=\bigcup_{I \subset\{1, \ldots, n\}} p^{I}\left(x_{1}, \ldots, x_{n}\right) \mathbb{Z}_{+}^{I},
$$

где $p^{I}(x)$ - моном следуюшего вида:

$$
p^{I}\left(x_{1}, \ldots, x_{n}\right)=\prod_{k \in I} x_{k} .
$$

ДокАЗАтЕльство. Полугруппа $\mathbb{Z}_{+}^{n}$ распадается в объединение $2^{n}$ непересекающихся подмножеств

$$
U_{I}=\left\{\left(m_{1}, \ldots, m_{n}\right) \mid m_{i}=0, i \notin I ; m_{i} \neq 0, i \in I\right\},
$$


где $I \subset\{1, \ldots, n\}$. Подмножество $U_{I}$ представляет собой координатную подполугруппу, сдвинутую на моном $p^{I}(x)$.

Теорема 1.8. Нормальная форма Т струи римановой метрики может быть выбрана такой, что метрические коэффициенты представляются в виде конечной суммь

$$
g_{i j}(x)=\delta_{i j}+\sum p_{i j}^{I}(x) \varphi_{i j}^{I}(x),
$$

где сумма берется по всем подмножествам $I \subset\{1, \ldots, n\}$, не содержащимся ни в одном из взаимно дополнительных подмножеств $I_{i j}=\{i, \ldots, j-1\}$ $u\{1, \ldots, n\} \backslash I_{i j}$, функциональный модуль $\varphi_{i j}^{I}(x)$ представляет собой формальный степенной ряд по переменным $x_{i}$, где $i \in I$, а моном $p_{i j}^{I}(x)$ имеет вид

$$
p_{i j}^{I}(x)=\prod_{k \in I} x_{k} .
$$

В частности, $|I| \geqslant 2 u \operatorname{deg} p_{i j}^{I} \geqslant 2$ для любого подмножества $I$, входячего в сумму.

ДокАЗАТЕльСтво. По теореме 1.5 нормальную форму Т можно выбрать так, что подпространства $\mathrm{T}_{i j}, 1 \leqslant i \leqslant j \leqslant n$, состоят из формальных бесконечных линейных комбинаций элементов подмножеств

$$
\mathrm{G}_{i j}=\mathbb{Z}_{+}^{n} \backslash\left(A_{i j} \cup B_{i j}\right) \subset \mathbb{Z}_{+}^{n},
$$

где по лемме 1.7 координатные подполугруппы $A_{i j}$ и $B_{i j}$ распадаются в объединение непересекающихся сдвинутых координатных подполугрупп:

$$
A_{i j}=\bigcup_{I \subset I_{i j}} p_{i j}^{I}(x) \mathbb{Z}_{+}^{I}, \quad B_{i j}=\bigcup_{I \subset\{1, \ldots, n\} \backslash I_{i j}} p_{i j}^{I}(x) \mathbb{Z}_{+}^{I} .
$$

Следовательно, подмножество $\mathrm{G}_{i j}$ представляется в виде

$$
\mathrm{G}_{i j}=\bigcup p_{i j}^{I}(x) \mathbb{Z}_{+}^{I},
$$

где $I \not \subset I_{i j}$ и $I \not \subset\{1, \ldots, n\} \backslash I_{i j}$.

Каждой сдвинутой координатной подполугруппе $p_{i j}^{I}(x) \mathbb{Z}_{+}^{I}$ в последнем разложении соответствует подпространство в $\mathrm{T}_{i j}$, состоящее из формальных бесконечных линейных комбинаций вида $p_{i j}^{I}(x) \varphi_{i j}^{I}(x)$, где $\varphi_{i j}^{I}(x)-$ формальный степенной ряд по переменным $x_{i}, i \in I$.

ОПРЕДЕЛЕНИЕ 1.9. Будем называть функциональные модули $\varphi_{i j}^{I}(x)$, входящие в нормальную форму, функщиональными модулями порядка $|I|$.

СлЕДСТВИЕ 1.10. Струю римановой метрики можно привести формальньлм диффеоморфизмом $к$ нормальной форме, содержащей конечное иисло функциональных модулей порлдка $|I| \geqslant 2$. В частности, существуют такие локальные координаты, в которых метрика имеет второй порядок касания с евклидовой метрикой.

В следующих примерах даны нормальные формы для двумерных и трехмерных струй римановых метрик. 
ПримеР 1. В случае струй римановых метрик на плоскости имеется единственный функциональный модуль $\varphi(x, y)$ порядка 2 . Следовательно, нормальная форма имеет вид $d s^{2}=d x^{2}+x y \varphi(x, y) d x d y+d y^{2}$, а подмножества $\mathrm{G}_{i j}$ в двумерном случае имеют следующий вид:

$$
\mathrm{G}_{11}=\mathrm{G}_{22}=\varnothing, \quad \mathrm{G}_{12}=\{(p, q) \mid p>0, q>0\},
$$

где $\mathrm{G}_{12}$ является координатной подполугруппой $\mathbb{Z}_{+}^{2}$, сдвинутой на моном $x y$.

ПримеР 2. В случае трехмерных струй римановых метрик подмножества $I_{i j} \subset\{1,2,3\}$ из теоремы 1.8 имеют вид $I_{12}=\{1\}, I_{13}=\{1,2\}, I_{23}=\{2\}$. Для каждого из них существует пара подмножеств мощности 2, не содержашихся ни в $I_{i j}$, ни в $\{1,2,3\} \backslash I_{i j}$ :

$$
\{1,2\} \text { и }\{1,3\} \text { для } I_{12}, \quad\{1,3\} \text { и }\{2,3\} \text { для } I_{13}, \quad\{1,2\} \text { и }\{2,3\} \text { для } I_{23} \text {. }
$$

Каждому из этих подмножеств соответствует функциональный модуль порядка 2 . Кроме того, все множество $\{1,2,3\}$ не содержится ни в одном из подмножеств $I_{i j}$. Следовательно, нормальная форма трехмерной струи римановой метрики имеет следующий вид:

$$
\begin{aligned}
g_{i i} & =1, \quad i=1,2,3, \\
g_{12} & =x_{1} x_{2} \varphi_{12}^{\{1,2\}}\left(x_{1}, x_{2}\right)+x_{1} x_{3} \varphi_{12}^{\{1,3\}}\left(x_{1}, x_{3}\right)+x_{1} x_{2} x_{3} \varphi_{12}^{\{1,2,3\}}\left(x_{1}, x_{2}, x_{3}\right), \\
g_{13} & =x_{1} x_{3} \varphi_{13}^{\{1,3\}}\left(x_{1}, x_{3}\right)+x_{2} x_{3} \varphi_{13}^{\{2,3\}}\left(x_{2}, x_{3}\right)+x_{1} x_{2} x_{3} \varphi_{13}^{\{1,2,3\}}\left(x_{1}, x_{2}, x_{3}\right), \\
g_{23} & =x_{1} x_{2} \varphi_{23}^{\{1,2\}}\left(x_{1}, x_{2}\right)+x_{2} x_{3} \varphi_{23}^{\{2,3\}}\left(x_{2}, x_{3}\right)+x_{1} x_{2} x_{3} \varphi_{23}^{\{1,2,3\}}\left(x_{1}, x_{2}, x_{3}\right) .
\end{aligned}
$$

Таким образом, в трехмерном случае имеется шесть функциональных модулей порядка 2 и три функциональных модуля порядка 3.

\section{§2. Ряд Пуанкаре пространства модулей струй римановых метрик}

ТЕОрема 2.1. Если геометрическал структура имеет нормальную форму с конечным числом функииональных модулей, то ряд Пуанкаре представляется следующей рачиональной функиией:

$$
p(t)=\sum_{q=0}^{n} \sum_{r=0}^{R} \frac{a_{r q} t^{r}}{(1-t)^{q}},
$$

где $n$ - размерность пространства, $a_{r q}$ - число функциональных модулей порядка $r$, зависящих от $q$ переменных, а $R$ - максимальный порядок функциональных модулей.

ДокАЗАТЕльство. Если нормальная форма имеет $m$ функциональных модулей $\varphi_{1}, \ldots, \varphi_{m}$ порядка $r$, зависящих от $q$ переменных, то размерность пространства орбит действия группы $\operatorname{Diff}\left(\mathbb{R}^{n}, 0\right)$ на пространстве $k$-струй равна числу мономов степени $k-r$ в формальных степенных рядах $\varphi_{1}, \ldots, \varphi_{m}$, а ряд Пуанкаре пространства струй такой геометрической структуры имеет вид

$$
p(t)=\frac{m t^{r}}{(1-t)^{q}} .
$$


В общем случае геометрической структуры с конечным числом функциональных модулей нужно просуммировать ряды такого вида, группируя функщиональные модули по порядкам и по числу переменных, от которых они зависят.

ЗАмЕчАниЕ 2.2. Набор функциональных модулей зависит от выбора способа приведения геометрической структуры к нормальной форме, в то время как ряд Пуанкаре инвариантен относительно такого выбора. Если выбрать другую нормальную форму с конечным числом функциональных модулей и применить к ней теорему 2.1, то ряд Пуанкаре, являющийся рациональной функцией, просто запишется в виде суммы других рациональных дробей.

ТЕОРема 2.3. Ряд Пуанкаре пространства модулей струй римановых метрик имеет вид

$$
p(t)=t^{2} q(t)+\sum_{r=2}^{n} \frac{a_{r} t^{r}}{(1-t)^{r}},
$$

где $q(t)$ 一нкоторый полином с неположительньми коэффичиентами,

$$
a_{r}=\frac{n(n-1)}{2}\left(\begin{array}{l}
n \\
r
\end{array}\right)-n \sum_{k=r}^{n-1}\left(\begin{array}{l}
k \\
r
\end{array}\right), \quad r=2, \ldots, n,
$$

a $n$ - размерность пространства. В частности, имеется $n(n-1) / 2$ функииональных модулей порядка $n$.

ДокАЗАТЕльство. Члену $g_{i j}, 1 \leqslant i<j \leqslant n$, нормальной формы Т соответствует подмножество $I_{i j} \subset\{1, \ldots, n\}, I_{i j}=\{i, \ldots, j-1\},\left|I_{i j}\right|=j-i$. Функциональные модули порядка $r$, входящие в $g_{i j}$, занумерованы $r$-подмножествами $I \subset\{1, \ldots, n\}$, такими, что $I \not \subset I_{i j}$ и $I \not \subset\{1, \ldots, n\} \backslash I_{i j}$. Число таких подмножеств равно

$$
b_{i j}=\left(\begin{array}{c}
n \\
r
\end{array}\right)-\left(\begin{array}{c}
j-i \\
r
\end{array}\right)-\left(\begin{array}{c}
n-j+i \\
r
\end{array}\right)
$$

где предполагается, что $\left(\begin{array}{l}p \\ q\end{array}\right)=0$ при $q>p$. Суммируя по всем $1 \leqslant i<j \leqslant n$, получаем

$$
a_{r}=\sum_{1 \leqslant i<j \leqslant n} b_{i j}=\frac{n(n-1)}{2}\left(\begin{array}{l}
n \\
r
\end{array}\right)-n \sum_{k=r}^{n-1}\left(\begin{array}{l}
k \\
r
\end{array}\right) .
$$

Поскольку нормальная форма, соответствующая трансверсали Т, представляет струи римановой метрики не однозначно, а лишь с точностью до некоторого конечномерного многообразия (см. замечание 1.4), то формальный степенной ряд рациональной функции

$$
\sum_{r=2}^{n} \frac{a_{r} t^{r}}{(1-t)^{r}}
$$


разложенный в нуле, еще не определяет ряда Пуанкаре пространства струй римановых метрик. Для получения ряда Пуанкаре необходимо к последнему формальному степенному ряду прибавить формальный степенной ряд $q(t)$ с неположительными коэффициентами, являющийся полиномом (так как трансверсаль Т пересекается с Diff $\left(\mathbb{R}^{n}, 0\right)$-орбитами по конечномерным многообразиям), причем в полиноме $q(t)$ отсутствуют члены нулевой и первой степени.

Например, при $n=2$ имеем $r=2$ и один функциональный модуль второго порядка, $a_{2}=1$. В этом случае ряд Пуанкаре имеет вид

$$
p(t)=t^{2} q(t)+\frac{t^{2}}{(1-t)^{2}}, \quad \text { где } q(t)=-t .
$$

При $n=3$ имеются функциональные модули второго и третьего порядка: $a_{2}=6, a_{3}=3$. В этом случае ряд Пуанкаре имеет вид

$$
p(t)=t^{2} q(t)+\frac{6 t^{2}}{(1-t)^{2}}+\frac{3 t^{3}}{(1-t)^{3}} .
$$

\section{ЛитературА}

1. Arnold V. I. Mathematical Problems in Classical Physics. Appl. Math. Sci., Vol. 100, Springer-Verlag, Berlin-New York, 1992.

2. Tresse A. Sur les invariants differentiels des groupes continus des transformations. Acta Math., 18 (1894).

3. Шмелев А. С. О дифференциальных инвариантах некоторых дифференциально-геометрических структур. Труды МИАН, 209, 234-267 (1995).

4. Кобаяси ШІ., Номидзу К. Основы дифференциальной геометрии. Т. 1, Наука, M., 1981. 